\title{
SFRP Synthesis of Acenaphthylene Oligomers and Block Copolymers. Potential Light Harvesting Structures
}

\author{
Dildar Ali, ${ }^{\dagger, \ddagger}$ Zaheer Ahmed, ${ }^{\S}$ Julian M. Dust, ${ }^{\#}$ Peter M. Kazmaier, ${ }^{\curvearrowleft}$ and Erwin Buncel ${ }^{\dagger, *}$ \\ ${ }^{\dagger}$ Department of Chemistry, Queen's University, Kingston, ON K7L 3N6, Canada. *E-mail: buncele@chem.queensu.ca \\ Institute of Biochemistry, University of Balochistan, Sariab Rd., Quetta, Pakistan \\ ${ }^{\S}$ H.E.J. Research Institute of Chemistry, University of Karachi, Karachi-75270, Pakistan \\ ${ }^{\#}$ Departments of Chemistry and Environmental Science, Grenfell Campus, Memorial University of Newfoundland, \\ Corner Brook, N.L. A2H 6P9, Canada \\ "The Xerox Research Centre of Canada, 2660 Speakman Drive, Mississauga, ON, L5K 2L1, Canada \\ Received March 2, 2011, Accepted May 31, 2011
}

\begin{abstract}
Azo-acenaphthylene oligomers with repeating acenaphthylene units " $\mathrm{n}$ " up to 4, 5, 7, 17 and 19 have been prepared successfully using nitroxide mediated Stable Free Radical Polymerization (SFRP). Azoacenaphthylene oligomers, reversibly end-capped by the stable nitroxide 2,2,6,6-tetramethyl-1-piperidinoxyl (TEMPO), were further reacted via radical addition to 4-(naphthalenemethoxy)styrene monomer for diblock co-polymer formation. Characterization of the oligomers and diblock co-polymers was accomplished using MALDI-MS supported by GPC (Gel Permeation Chromatography) and ${ }^{1} \mathrm{H}$ NMR spectrometry. MALDI-MS afforded definitive results by providing an inter-peak interval of $152(\mathrm{~m} / \mathrm{z})$, corresponding to acenaphthylene monomer, and inter-peak interval of $260(\mathrm{~m} / \mathrm{z})$ for the naphthalenemethoxystyrene monomer unit in block copolymers. Our study opens the way to control the number of repeat units in the oligomers. Further these oligomers can be tailored with various monomers for the formation of block copolymers.
\end{abstract}

Key Words : Acenaphthylene, Block, Oligomers, SFRP

\section{Introduction}

Considerable research effort continues to be focused on the investigation and development of light harvesting (LH) systems, where appropriately constructed macromolecules serve to mimic aspects of photosynthesis. ${ }^{1-7}$ Part of our ongoing research in the area of materials science ${ }^{8-11}$ involves investigation of the underlying processes of light harvesting, including absorption of light by suitable donor (D) moieties and efficient energy (and/or) electron transfer to acceptor (A) moieties in LH model molecules.

In our studies we found, in particular, ${ }^{11}$ that the efficiency of energy transfer (ET) is controlled by the specific molecular architecture linking D and A units in the LH model system. This is illustrated in Scheme 1: Donor D groups absorb light energy which is then transferred from the excited D $\left(D^{*}\right)$ to the acceptors A; D groups in the case of

Model A

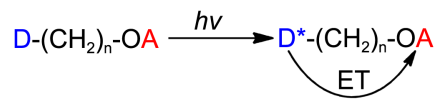

Model B

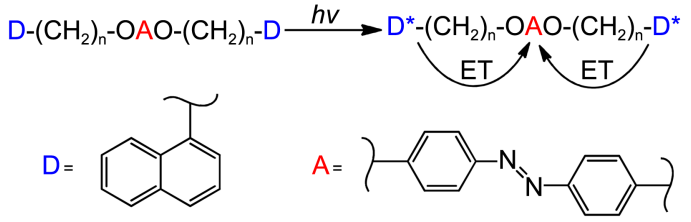

Scheme 1 polymers and particularly peripherally-substituted starburst or dendrimeric polymers, have been likened to "antennae."12

Various strategies have been used to approach controlled radical polymerization to give well-defined structure, ${ }^{13-16}$ including Atom Transfer Radical Polymerization (ATRP), ${ }^{17}$ Radical Addition Fragmentation (RAFT), ${ }^{18-20}$ and Nitroxide Mediated Polymerization (NMP) ${ }^{13}$ also termed Stable Free Radical Polymerization (SFRP).

The SFRP approach developed in our work (see Scheme 2 ) is based on unimer $\mathbf{1}$ as initiator; this includes an azobenzene acceptor group and is end-capped with the labile TEMPO (2,2,6,6-tetramethyl-1-piperidinoxyl) group. Heating of this unimer releases the persistent nitroxyl TEMPO and the reactive oligomer end-radical, and this radical adds to a different vinylic monomer leading to diblock copolymer formation.

We made successful use of the SFRP approach recently to synthesize a range of azo-acetoxystyrene and azo-naphthalene oligomers ${ }^{13}$ with varying molecular weights (Scheme 2 ). These potential LH oligomers, derived from the azounimer 1, with repeating maximum $n$ values ranging from 2 to 52 , were synthesized.

The current extension of the work to the preparation of oligomers containing the azo-benzene Acceptor and acenaphthylene Donor was of interest to ascertain whether the more rigid acenaphthylene structure would change energy transfer properties involving this moiety noting that bond rotation normally enables energy loss from the excited state through rotational coupling. Our specific interest lies 


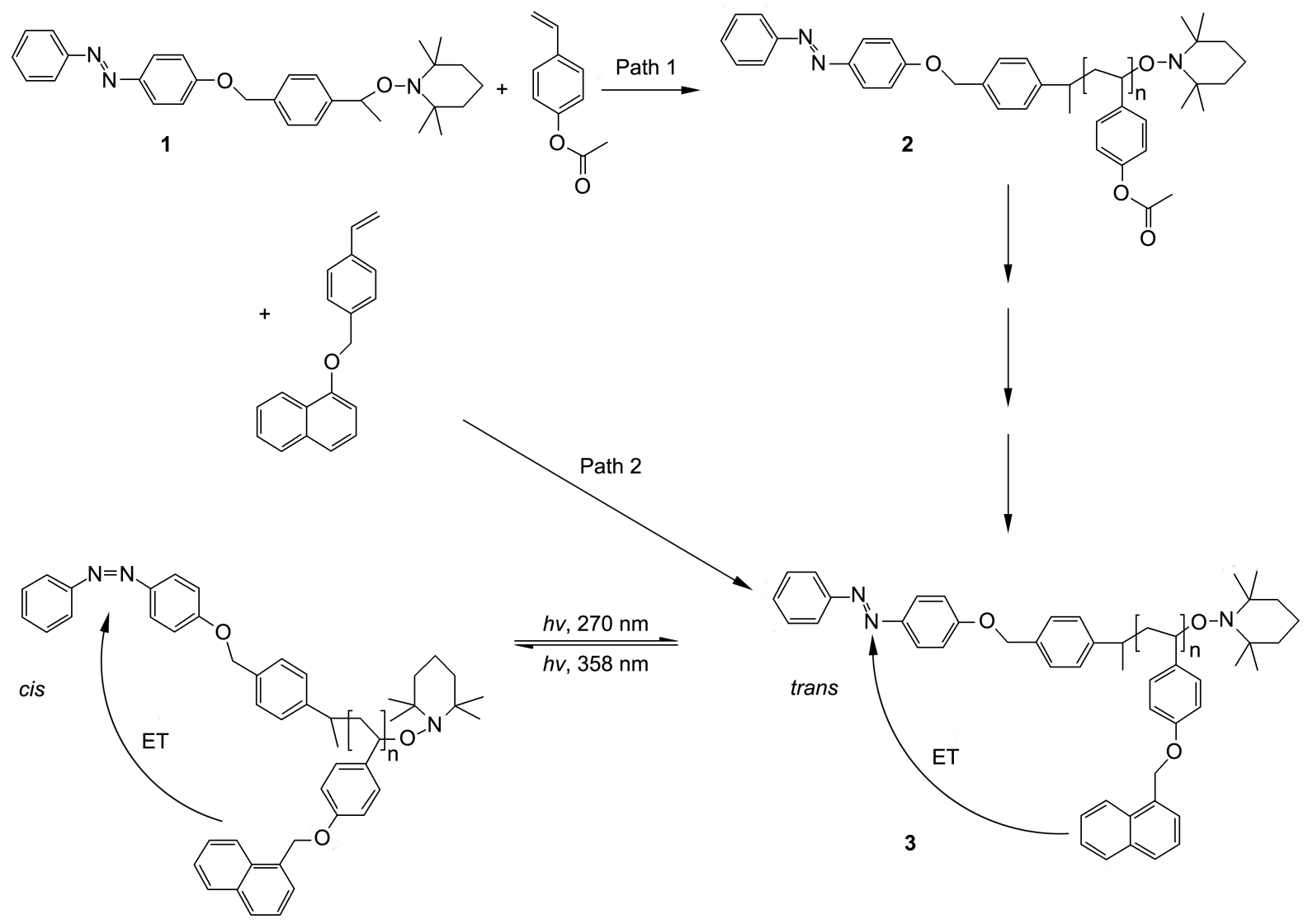

Scheme 2

in preparation of suitably end-substituted oligomers for potential use as light harvesting models. In a separate preparation, the oligomeric azobenzene-containing acenaphthylene oligomer was then reacted with 4-(naphthalenemethoxy)styrene as the vinyl monomer to give a diblock copolymer. Looking further ahead, could one induce chirality, for example, by polymerizing acenaphthylene using a chiral nitroxide? Different approaches of this kind would become available with development of this work.

Thus, in the current paper we report on the synthesis of acenaphthylene oligomers; further, these oligomers have been elaborated by chain extension with naphthalenemethoxystyrene which has led to the preparation of the block copolymers.

\section{Experimental}

\section{Instruments and Methods.}

${ }^{1}$ H NMR: ${ }^{1} \mathrm{H}$ NMR spectra were recorded on a Bruker Avance-400 automatic spectrometer operating at $400.1 \mathrm{MHz}$ $\left({ }^{1} \mathrm{H}\right)$. All chemical shifts are reported as $\delta$ in parts per million (ppm) relative to the peaks for residual $\mathrm{CHCl}_{3}$ in $\mathrm{CDCl}_{3}(\delta=$ 7.28) or residual $\mathrm{CH}_{2} \mathrm{Cl}_{2}$ in $\mathrm{CD}_{2} \mathrm{Cl}_{2}(\delta=5.30)$. Coupling constants $(\mathrm{J})$ are reported in Hertz $(\mathrm{Hz})$.

Gel Permeation Chromatography (GPC): GPC was performed using a Waters 2695 Gel Permeation Chromatograph equipped with a Waters 410 differential refractometer set to $40{ }^{\circ} \mathrm{C}$, separation module with four Waters Ultrastyragel columns (HR5.0, HR3.0, HR1.0, and HR0.5) in series. Distilled tetrahydrofuran was used as the eluent at a

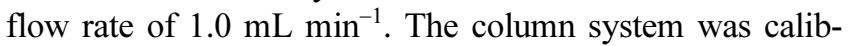
rated with polystyrene standards.

MALDI-MS: MALDI-MS was performed on Applied Biosystems Voyager DE-STR MALDI-time of flight-mass spectrometer. This instrument is equipped with a nitrogen laser $(337 \mathrm{~nm})$, delayed extraction and reflector. It was operated at an accelerating potential of $20 \mathrm{kV}$ in both linear and reflector mode. The 2,5-dihydroxybenzoic acid (DHB) matrix was used without added salt for oligomer characterization to selectively protonate the alkoxyamine (TEMPO) moiety.

Sample Preparation for MALDI-MS: ${ }^{24}$ The oligomeric samples were prepared by mixing a solution of oligomers in THF $(1 \mathrm{mg} / \mathrm{mL})$, and a solution of DHB matrix $(20 \mathrm{mg} / \mathrm{mL})$ in 1:1 ratio of acetonitrile and $\mathrm{MeOH}$ to give the analytical matrix. After mixing analyte and matrix $\left(\mathrm{V}_{\text {sample }} / \mathrm{V}_{\text {matrix }}\right)$ (1:1), $1 \mu \mathrm{L}$ of this mixture was placed onto the sample plate and the solvent evaporated at room temperature to give the MALDI-MS sample for characterization.

General Experimental Procedure for the Synthesis of Oligomers 4 and Block Copolymers 5.

Materials: All common solvents (acetone, THF, $\mathrm{MeOH}$ etc.) used in the preparations outlined below were purchased commercially in HPLC grade or better and, where necessary, 
were further purified by standard methods. ${ }^{25}$ All inorganics and the remaining requisite chemicals and organic reagents were purchased commercially and used without further purification.

The azo unimer 1 was prepared in a five-step procedure as previously described $^{8,13}$ The vinyl monomer used in the copolymerization studies, 4-naphthalenemethoxystyrene, was prepared as previously reported by us. ${ }^{13}$ The 2,2,6,6-tetramethyl-1-piperidinoxyl (TEMPO, gift of Xerox Research Canada) was used as received.

Synthesis of Acenaphthylene Oligomers, 4 - A mixture of azobenzene unimer 1, acenaphthylene and chlorobenzene (proportions given in Table 1) were taken in a round bottom flask (RBF), sealed with rubber septum and purged with $\mathrm{N}_{2}$ for $5 \mathrm{~min}$, immersed into a preheated, stabilized oil bath at $120{ }^{\circ} \mathrm{C}$ and reaction allowed to take place. After the set reaction times, addition of cold methanol $(10 \mathrm{~mL})$ terminated reaction along with precipitation of the oligomeric product; filtration was followed by drying in vacuo $(<1$ Torr, $50{ }^{\circ} \mathrm{C}$ ) overnight to give brown powders of 4 . The products were characterized by MALDI-MS supported by GPC and ${ }^{1} \mathrm{H}$ NMR spectrometry.

Synthesis of Block Copolymers, 5-In Scheme 4, the short chain block copolymers containing acenaphthalene (n) and 4-(naphthalenemethoxy)styrene (m) repeating units were prepared using nitroxide mediated polymerization in $\mathrm{PhCl}$ at $120{ }^{\circ} \mathrm{C}$, where 4 acted as the initiator for the addition polymerization of the 4-(naphthalenemethoxy)styrene monomer. In one experiment a mixture of acenaphthylene oligomer 4 (22.1 mg, $0.0085 \mathrm{mmol}$ ) with maximum $n$ value of 17 (Experiment D, Table 1) and 4-(naphthalenemethoxy)styrene monomer (40 mg, $0.153 \mathrm{mmol})$ in $1 \mathrm{~mL}$ of $\mathrm{PhCl}$ was taken in a round bottom flask. The RBF was sealed with a Teflon rubber septum and purged with $\mathrm{N}_{2}$ for $5 \mathrm{~min}$, then immersed in an oil bath at $120^{\circ} \mathrm{C}$. An aliquot of reaction mixture was taken after $20 \mathrm{~min}$ and poured into copious cold methanol $(10 \mathrm{~mL})$ to terminate the reaction and the rest of the mixture was heated for an additional $30 \mathrm{~min}$ (total 50 $\mathrm{min})$. After $50 \mathrm{~min}$ the reaction was quenched with cold $\mathrm{MeOH}(10 \mathrm{~mL})$. The resulting crude products 5 precipitated upon addition of methanol and were then filtered off, dried as above and characterized by MALDI-MS along with GPC, ${ }^{1} \mathrm{H}$ NMR spectrometry.

\section{Results and Discussion}

Our strategy uses the approach of controlled polymerization via SFRP in conjunction with azo-unimer $\mathbf{1}^{8,11,13}$ containing an azo functionality (a suitable acceptor moiety for LH studies) and the TEMPO (2,2,6,6-tetramethylpiperidinyl-1-oxyl) moiety for reversibly capping the growing chain with acenaphthylene (AcNp) monomer. Chlorobenzene $(\mathrm{PhCl})$ is added as a diluent to suppress self-polymerization of $\mathrm{AcNp}$, as well as to control molecular weight (MW) and polydispersity $\left(\mathrm{PD}=\mathrm{M}_{\mathrm{w}} / \mathrm{M}_{\mathrm{n}}\right)$, of the growing oligomer, 4 (eq. (1)).

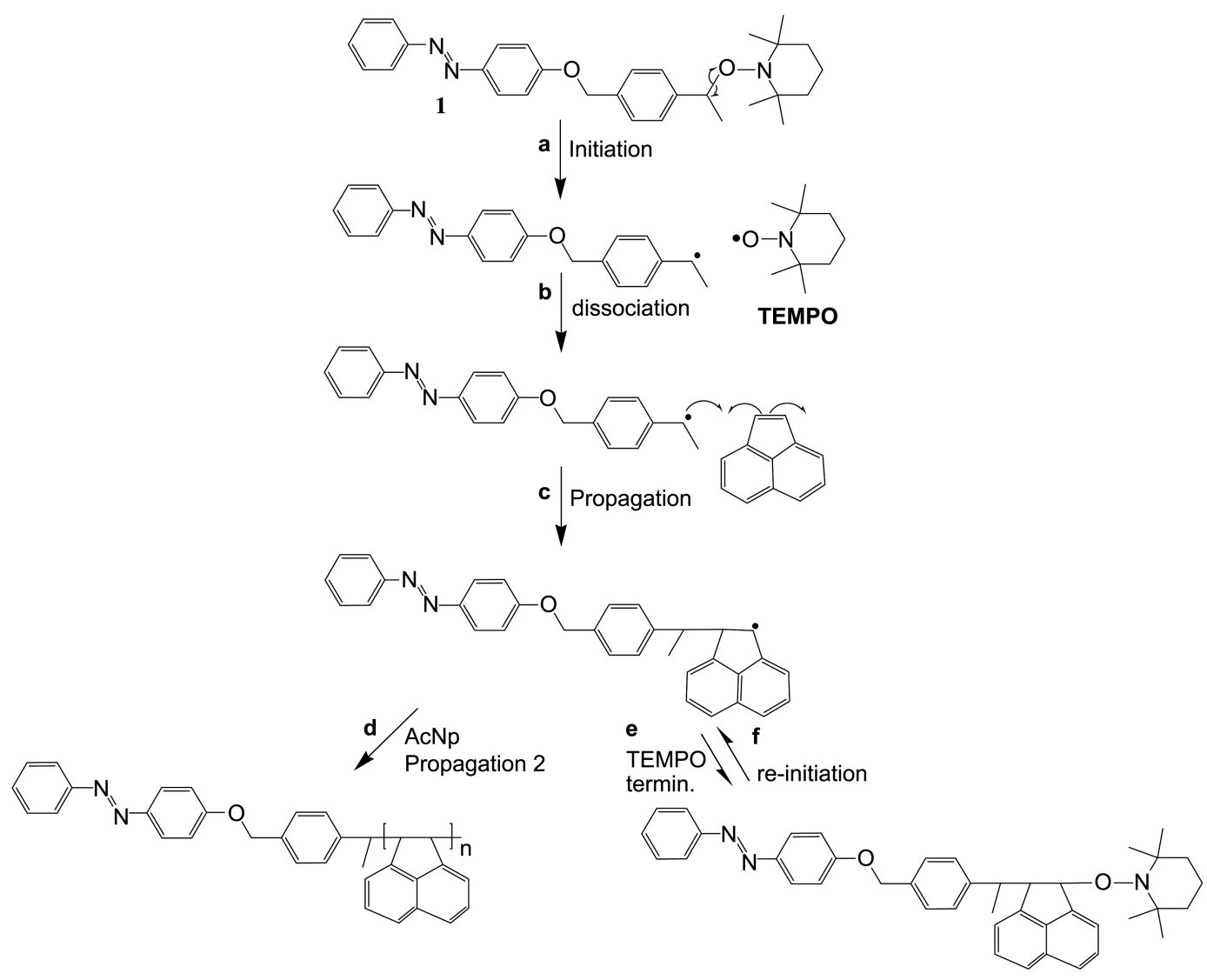

Scheme 3 


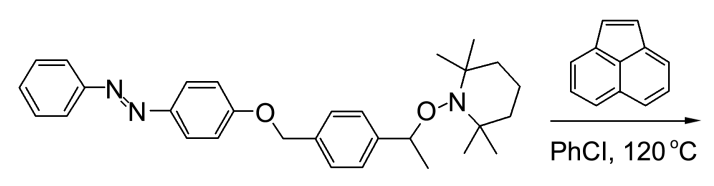

1

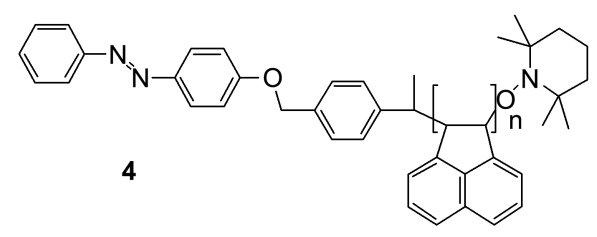

Mechanistically, at $120^{\circ} \mathrm{C}$, the alkoxyamine C-O bond that links the TEMPO cap to the benzylic site of $\mathbf{1}$ cleaves in the initiation step ( $a$, Scheme 3 ). This leads to formation of a solvent caged radical pair, consisting of a resonance stabilized benzylic radical $^{26}$ and the persistent TEMPO nitroxyl radical. Dissociation ( $b$, Scheme 3 ) of the radical pair results in addition to the non-aromatic (vinyl-like) double bond of acenaphthylene (AcNp) in a propagation step ( $c$, Scheme 3). The newly-formed benzylic radical may now partition between further addition in another propagation step ( $d$, Scheme 3), extending the oligomer chain or coupling with TEMPO in a termination step ( $e$, Scheme 3$)$. Fragmentation of the TEMPO-capped (dead) oligomer reinitiates the oligomerization ( $f$, Scheme 3 ). It is the balance in this partitioning that is generally considered to give oligomers/polymers of relatively low polydispersity. The overall process is shown in eq. (1).

Acenaphthylene Oligomers, 4. To synthesize a range of low molecular weight azo-acenaphthylene oligomers 4 under controlled conditions the proportion of the respective components $(\mathbf{1}, \mathrm{AcNp}, \mathrm{PhCl})$ and the reaction time, were varied as shown in Table 1 , experiments $A$ to $E$. The optimized conditions given in Table 1 minimized thermal self-polymerization of AcNp.

Addition of chlorobenzene $(\mathrm{PhCl})$ solvent could have two effects. ${ }^{14,15}$ The first and presumably smaller effect is a solvent effect on the dissociation of the unimer 1 (a, Scheme 3). Preferential solvation of the dissociated radicals (e.g. via $\pi-\pi$ stacking) relative to the bulky TEMPO-capped unimer could favour fragmention of the unimer 1 into the respective benzylic and TEMPO radicals. On the other hand, the concentration of AcNp in the cage surrounding the radical

Table 1. Azo-acenaphthylene oligomers 4 synthesis: reaction conditions for the oligomerization

\begin{tabular}{cccccc}
\hline Exp & Azo unimer 1 & AcNp & PhCl & Time & MALDI-MS \\
\hline & $\mathrm{mg}(\mathrm{mmol})$ & $\mathrm{mg}(\mathrm{mmoL})$ & $(\mathrm{mL})$ & $(\mathrm{min})$ & $(n)^{a}$ \\
\hline $\mathrm{A}$ & $5(0.01)$ & $40(0.26)$ & 0.5 & 20 & 4 \\
$\mathrm{~B}$ & $10(0.02)$ & $40(0.26)$ & 1 & 60 & 5 \\
$\mathrm{C}$ & $12(0.025)$ & $50(0.32)$ & 1 & 60 & 7 \\
$\mathrm{D}$ & $15(0.031)$ & $100(0.64)$ & 1 & 50 & 17 \\
E & $37(0.082)$ & $136(0.90)$ & 1.5 & 80 & 19
\end{tabular}

${ }^{a}(n)$ is the highest number of repeating acenaphthylene units observed in MALDI-MS spectra of $\mathbf{4}$ pair would be reduced as a function of increasing added $\mathrm{PhCl}$ and the probability of addition to the double bond of AcNp diminished. At short reaction times the number of repeating groups incorporated in the oligomer would be reduced, as is observed (Table 1). As seen, up to four repeating units are incorporated with $20 \mathrm{~min}$ reaction time and $0.5 \mathrm{~mL}$ of $\mathrm{PhCl}$ (exp. A). A longer reaction time of 60 min (exp. B) is mitigated by an increase in $\mathrm{PhCl}$ diluent to 1 $\mathrm{mL}$, resulting in oligomer with a maximum $n=5$. Finally, at 80 min reaction time (exp. E) $n$ has reached 19 repeating units. Thus, there is an interplay of reaction time and amount of $\mathrm{PhCl}$ diluents used.

As shown in Table 1, acenaphthylene oligomers, 4, ranging with maximum repeating number $n$ acenaphthylene monomer units between 4 and 19 were prepared. Characterization of $\mathbf{4}$ was accomplished using a number of techniques: MALDI-MS, GPC and ${ }^{1} \mathrm{H}$ NMR spectrometry.

Characterization of Azo-acenaphthylene Oligomers 4:

Oligomer Analysis by MALDI-MS - In Figure 1 are given representative MALDI-MS spectra for $\mathrm{A}, \mathrm{B}$, and $\mathrm{C}$ experiments (Table 1). Molecular weights are calculated for oligomers with each increasing maximum number of repeat units " $n$ ". The azo part (AZ) represents a molecular weight of $316(\mathrm{~m} / \mathrm{z})$, one acenaphthylene monomer $(\mathrm{AcNp})$ molecular weight is equal to $152(\mathrm{~m} / \mathrm{z})$ and TEMPO (T) has the molecular weight of $155(\mathrm{~m} / \mathrm{z})$. These weights are added up for various $n$ values (i.e., $n$ AcNp repeating units) and sample calculations are shown in Figure 1. The MALDI-MS spectrum of azo-acenaphthylene oligomer with $n$ up to 4 is shown in Figure 1 (Experiment A). The peak at $[\mathrm{M}+\mathrm{H}]^{+}=$ $1080 \mathrm{~m} / \mathrm{z}$ corresponds to a maximum of 4 repeating units. Peaks due to successive loss of 152 mass units corresponding to acenaphthylene repeating units are highlighted. Comparable results were obtained for other oligomers (B to E) as given in Table 1. These MALDI-MS peaks are reported as relative intensities with an estimated $\pm 5-10 \%$ error.

Careful examination of the MALDI-MS traces recorded for samples prepared with longer reaction times (i.e. Fig. 1, traces b and c) included small peaks at $m / z$ values greater than those set by sequential loss of acenaphthylene repeating units alone. For example, in Figure 1(a) the $1080 \mathrm{~m} / \mathrm{z}$ peak ascribable to oligomer with up to 4 repeating units is relatively clean with only negligible signals at higher $\mathrm{m} / \mathrm{z}$ values. The same $n=4$ peak in Figure 1(b and c) is accompanied by signals at 1098 (i.e., $n=4+18.01 \mathrm{~m} / \mathrm{z}$ ) and 1114 $\mathrm{m} / z$ (i.e. $1098 \mathrm{~m} / \mathrm{z}+16.00 \mathrm{~m} / \mathrm{z}$, particularly notable in Figure $1(c))$. These signals could indicate the incorporation of water (1098 $\mathrm{m} / \mathrm{z}, 18.01 \mathrm{~m} / \mathrm{z}$ above $n=4)$ and oxygen $(1114 \mathrm{~m} / \mathrm{z}$, $16.00 \mathrm{~m} / z$ above the $1098 \mathrm{~m} / \mathrm{z}$ peak). As such, the 1098 and $1114 \mathrm{~m} / \mathrm{z}$ signals could arise from oxidative side-reactions significant only at longer reaction time (see Table 1). Alternatively, oligomer structural defects arising from radical addition at other sites (as a result of spin delocalization in the two benzyl-type radicals, ${ }^{26}$ Scheme 3 ) could lead to alternative fragmentation pathways giving rise to these and other small peaks, particularly in higher molecular weight product. Nonetheless, these MALDI-MS results clearly show that by 

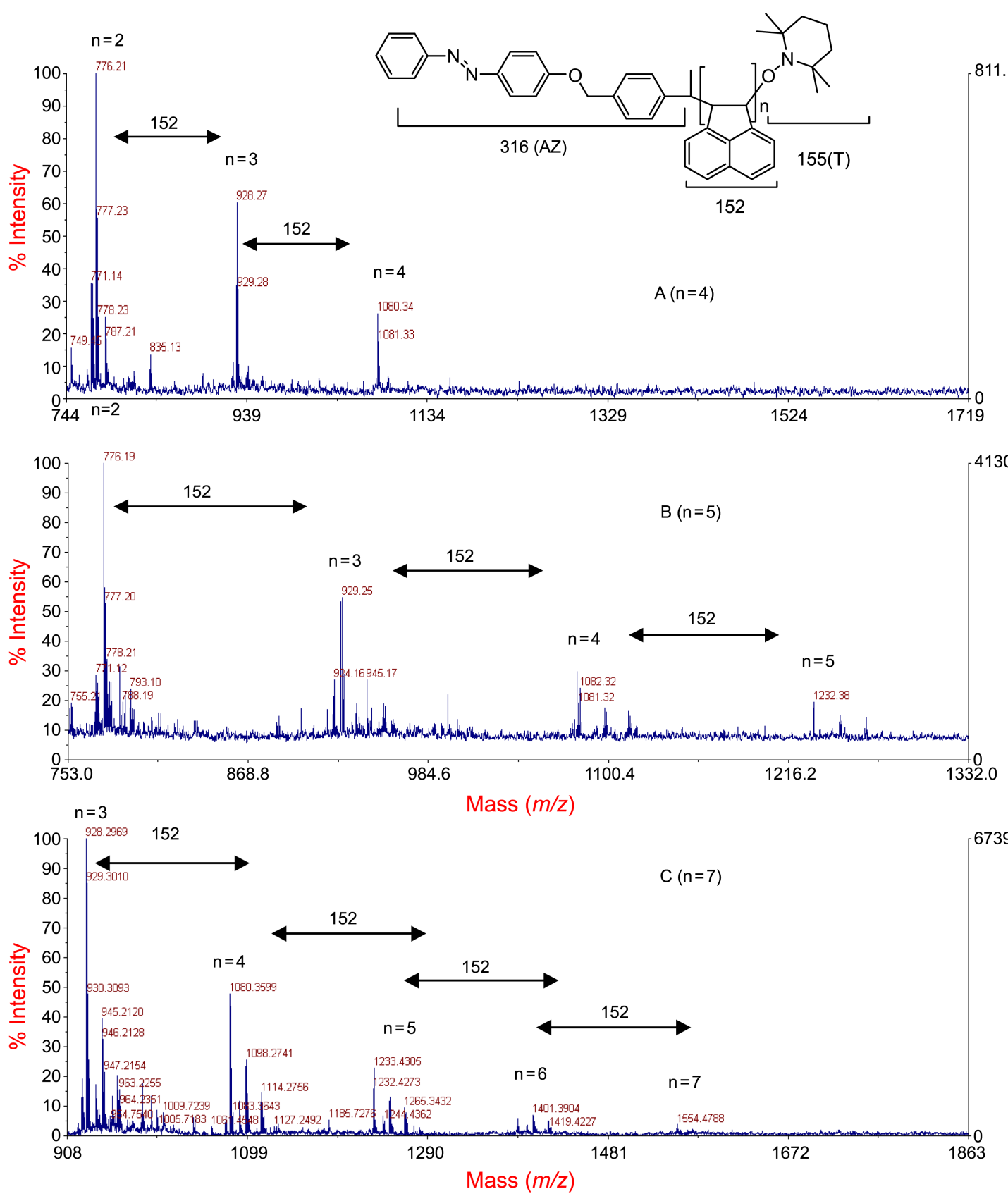

Figure 1. MALDI-MS spectra of acenaphthylene oligomers (A) with $n$ up to 4 , (B) $n$ maximum $=5$ and (C) $n$ up to 7 . The inter-peak interval is $152(\mathrm{~m} / \mathrm{z})$ which reflects the mass of the acenaphthylene repeat unit. Therefore, calculation of molar mass for $(\mathrm{A})$ is: $[\mathrm{M}+\mathrm{H}]^{+}$at $1080=316($ Azo $)+4 \times 152(\mathrm{AcNp})+155(\mathrm{~T})+1(\mathrm{H})$. Similar calculation formula applies for all $n$ values.

manipulating reaction conditions (Table 1) a range of azoacenaphthylene oligomers, $\mathbf{4}$, has been successfully prepared using the SFRP methodology. Thus MALDI MS highlights the sequential loss of acenaphthylene units, minus $152 \mathrm{Da}$, and clearly confirms the maximum number of repeating units in each sample of oligomer 4.

The following GPC (Gel Permeation Chromatography) and ${ }^{1} \mathrm{H}$ NMR results provide corroborating evidence.

Oligomer Analysis by GPC - Data from GPC chromatograms (Figure 1S for experiments A-D in Supplementary Information) for experiments $\mathrm{A}$ through $\mathrm{E}$ are collected in Table 2. In Table 2 both number average molecular weights
Table 2. GPC results for azo-acenaphthylene oligomers, 4

\begin{tabular}{ccc}
\hline Experiment & $\mathrm{M}_{\mathrm{n}}$ & P.D. \\
\hline $\mathrm{A}$ & 500 & 1.35 \\
$\mathrm{~B}$ & 810 & 1.30 \\
$\mathrm{C}$ & 560 & 1.32 \\
$\mathrm{D}$ & 1140 & 1.63 \\
$\mathrm{E}$ & 1010 & 1.16 \\
\hline
\end{tabular}

${ }^{a} \mathrm{GPC}$ column system calibrated with polystyrene standards.

$\left(\mathrm{M}_{\mathrm{n}}\right)$ and polydispersity (P.D. $\left.=\mathrm{M}_{\mathrm{w}} / \mathrm{M}_{\mathrm{n}}\right)$ values are given, determined using polystyrene standard calibration curves. In 
each trace a peak attributed to unmodified $\mathbf{1}$ is present (Figure 1S).

As in the results of MALDI-MS where experiments A-E show increasing values of $n$, GPC shows a rough trend to increasing $\mathrm{M}_{\mathrm{n}}$ along the series. The P.D. values generally fall in the region of relatively low polydispersity for these types of polymers produced by a free radical mechanism (i.e. $<1.5)$. Altogether the GPC results though not definitive are consistent with the MALDI-MS results.

Oligomer Analysis by ${ }^{1} \mathbf{H}$ NMR Spectrometry - The ${ }^{1} \mathrm{H}$ NMR spectra (400 MHz, Figure 2S in Supplementary Information) show characteristic peaks in the aromatic region 6.9-8.2 ppm corresponding to $\mathrm{H}_{1}-\mathrm{H}_{12}$ for the azobenzene and phenyl moieties, and $\mathrm{H}_{27-33}$ for the $\mathrm{AcNp}$ repeat units; aliphatic protons $\mathrm{H}_{14-24}$ are found at $0.6-2.0 \mathrm{ppm}$. As the maximum number of repeating acenaphthylene monomer units $n$ increases, broadening of signals in both the aromatic and the aliphatic region increases and is in accordance with formation of higher molecular weight oligomers, as shown through MALDI-MS and GPC results.

Altogether consistent structural evidence is obtained for oligomers, 4, with increasing $n$ values over the range 4-19.

Block Copolymers 5. Block copolymers are composed of "blocks" of the same monomer units covalently bound to sequences of unlike type and these may be prepared using the SFRP strategy. ${ }^{27,28}$ In the present studies, we have synthesized linear homo azo-acenaphthalene oligomers 4 with various repeating units $n$ and then extending to block copolymer formation. We have used acenaphthylene oligomers $\mathbf{4}$ as initiators for formation of block copolymers using a vinyl monomer containing a naphthalenic moiety, 4(naphthalenemethoxy)styrene (m, Scheme 4). Note for potential LH studies the naphthalenic moiety would function as the Donor group (Scheme 1) in the resulting block copolymer. Various combinations of oligomers 4 and 4(naphthalenemethoxy)styrene are observed in MALDI-MS (See Table 3 and Fig. 2). GPC data supports formation of the diblock co-polymer, 5 (Fig. 3S in Supplementary Information), as does ${ }^{1} \mathrm{H}$ NMR spectrometry (Fig. 4S).

Characterization of Diblock Copolymers, 5:

MALDI-MS Analysis of 5-Co-polymers in general exhibit more complex MALDI-MS spectra due to the various possible combinations of repeating units. In Table 3 are provided the molar masses for a few combinations of repeating units (n) and (m, see Scheme 4) which are obtained through analysis of Figure 2. Here the MALDI-MS spectra characterize the diblock copolymer arising from reaction of the acenaphthylene oligomer $(n=17)$ with 4-(naphthalenemethoxy)styrene with $20 \mathrm{~min}$ reaction time (A), and $50 \mathrm{~min}$ reaction time (B). Spectrum (A) shows clearly the sequential loss of the $152 \mathrm{Da}$ repeating unit corresponding to the acenaphthylene moiety. A growing complex peak pattern corresponding to the 4-(naphthalenemethoxy)styrene repeating unit is also seen in (B), which on expansion in $(\mathbf{C})$ displays clearly the $260 \mathrm{Da}$ for the sequential cleavage of this repeating unit from the diblock copolymer, $\mathbf{5}$, thus providing definitive evidence for diblock copolymer formation.

Diblock Copolymer Analysis by GPC - Comparative GPC traces (not shown) support formation of diblock copolymers 5 (Scheme 4) in broad agreement with MALDIMS results. Generally, the peak for the copolymer appears at shorter retention times than that of residual starting oligomer $\mathbf{4}$, in accord with the higher molecular weights of $\mathbf{5}$ with

Table 3. MALDI-MS results for diblock copolymers, 5

\begin{tabular}{ccc}
\hline$(\mathrm{n}),(\mathrm{m})^{a}$ & Experimental mass & Calculated mass \\
\hline 1,2 & 1143 & 1144.60 \\
1,3 & 1403 & 1404.71 \\
1,4 & 1664 & 1664.83 \\
2,2 & 1296 & 1295.65 \\
2,3 & 1556 & 1555.77 \\
2,4 & 1816 & 1816.90 \\
2,5 & 2076 & 2077.02 \\
3,3 & 1708 & 1708.84 \\
4,3 & 1860 & 1860.90 \\
4,4 & 2120 & 2121.02 \\
5,1 & 1491 & 1492.72 \\
5,2 & 1752 & 1752.84 \\
5,3 & 2011 & 2012.96 \\
\hline
\end{tabular}

${ }^{a}$ The designations $\mathbf{n}$ and $\mathbf{m}$ refer to the number of acenaphthylene and naphthalenemethoxystyrene units, respectively, in copolymer $\mathbf{5}$.

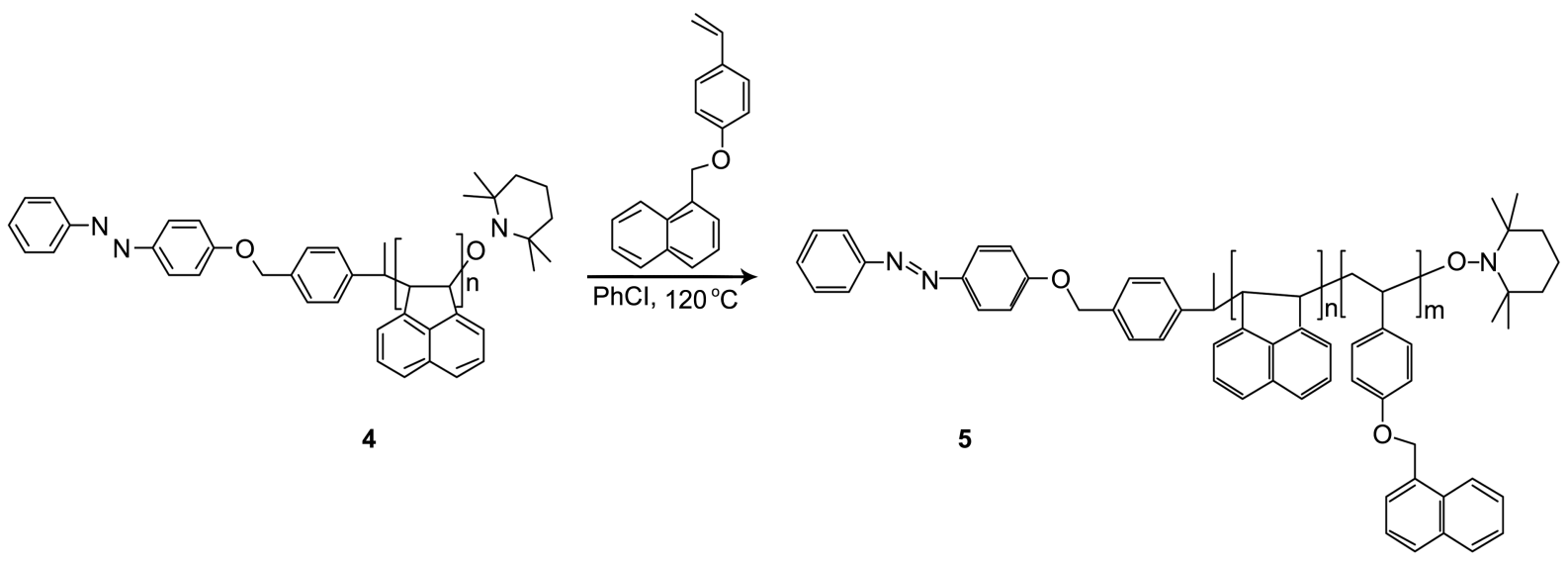



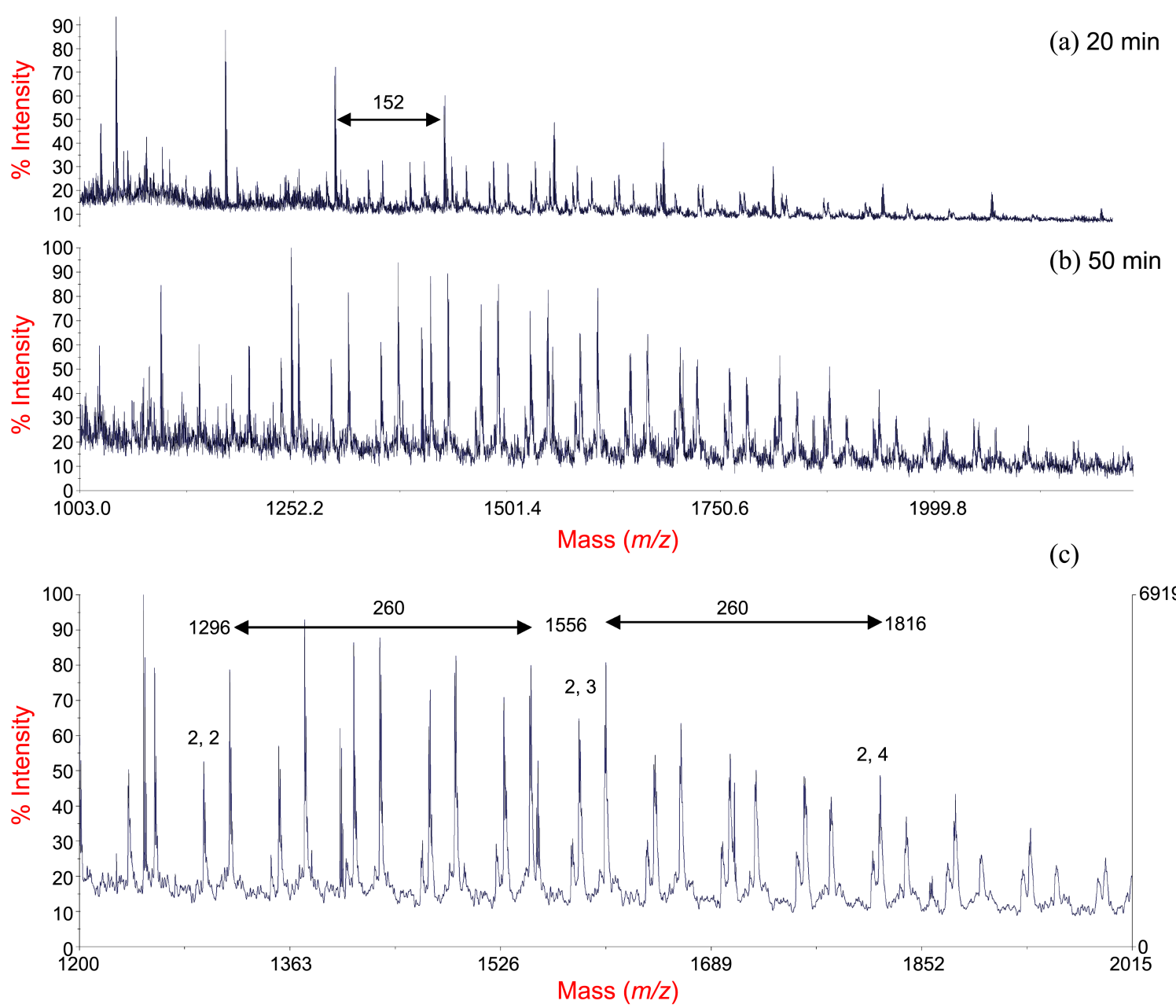

Figure 2. MALDI-MS spectra for the diblock copolymer 5 formed at $20 \mathrm{~min}$. reaction time (a, top) and at 50 min reaction time (b). The bottom spectrum (c) is an expansion of the 1200 to $2015 \mathrm{~m} / \mathrm{z}$ region of B showing sequential loss of the 4-(naphthalenemethoxy)styrene repeating unit $(260 \mathrm{~m} / \mathrm{z})$ from $\mathbf{5}$.

respect to 4 . The increased broadness of the GPC peak for the diblock copolymer formed with 50 min reaction time compared to the peak found at 20 min of reaction translates into increasing P.D. with increasing reaction time.

The lack of a suitable calibration standard for 5 precludes assignment of $M_{\mathrm{n}}$ and P.D. values by GPC in these systems. Nonetheless, the GPC results provide qualitative evidence of the formation of $\mathbf{5}$ with varying molecular weights in accord with the more quantitative MALDI-MS results.

Diblock Copolymer Analysis by ${ }^{1} \mathrm{H}$ NMR Spectrometry $-{ }^{1}$ H NMR spectra (400 MHz) were recorded (Figure 4S) for the initial oligomer $\mathbf{4}$ and for the diblock copolymers $\mathbf{5}$ formed by addition of $\mathbf{4}$, as an initiator, to 4 -(naphthalenemethoxy)styrene, a vinyl monomer, at $20 \mathrm{~min}$ and $50 \mathrm{~min}$ reaction times, respectively. While the aromatic protons of both 4 and 5 largely overlap in the downfield $(\delta 6.2-8.0)$ region and similarly most aliphatic protons overlap in the upfield (0.6-1.8 ppm) region of the spectra, unique diagnostic signals can be discerned. In the upfield region a somewhat broadened "singlet" appears at $\delta 2.0$. Although not conclusively identified it likely arises from the benzylic methine of $\mathbf{4}$ ( $\mathrm{CH}$ attached to the methyl labelled "e" in Fig.
$4 \mathrm{~S}$ ), where coupling is unresolved. Regardless, the signal belongs to 4 . This signal is visible at diminished intensity in the spectrum of the copolymer $\mathbf{5}$ (20 min reaction), as expected for a "dilution" of the original oligomer $\mathbf{4}$ as a component of the diblock copolymer $\mathbf{5}$. The signal is no longer readily visible in the spectrum of the higher molecular weight 5 (50 min reaction time). In fact, all signals in the aliphatic region diminish in intensity relative to those of the aromatic region for the copolymer $\mathbf{5}$ as would be expected since the 4-(naphthalenemethoxy)styrene repeating units in 5 contain predominantly aromatic protons.

Equally important is the observation of the methoxy methylene of the 4-(naphthalenemethoxy)styrene repeating unit (i.e. Np-CH $\boldsymbol{H}_{2}-\mathrm{O}-$, labelled "i" in Fig. $4 \mathrm{~S}$ ) as a sharp singlet at $5.0 \mathrm{ppm}$. Understandably, this singlet is not present in the spectrum of $\mathbf{4}$, but is seen in both spectra of 5 appearing at greater intensity in the higher molecular weight (50 min reaction) copolymer $\mathbf{5}$. Further, the spectra of $\mathbf{5}$ contain a multiplet at $c a .8 .0 \mathrm{ppm}$ ascribable to an aromatic proton(s) (not assigned) in the 4-(naphthalenemethoxy)styrene repeating unit; the multiplet is not present in the spectrum of $\mathbf{4}$ and thus provides further evidence for 
formation of $\mathbf{5}$ under both reaction conditions. Increasing broadness in the aliphatic and aromatic regions, generally, is also noted as a function of increasing numbers of repeating units in $\mathbf{5}$ in agreement with the quantitative MALDI-MS results. Thus despite the complexity of the ${ }^{1} \mathrm{H}$ NMR spectra the analysis provided is fully in accordance with diblock copolymer formation through reaction of 4 with 4-(naphthalenemethoxy)styrene as shown in Scheme 4.

\section{Conclusion}

As part of a long-term program to investigate light harvesting (LH) structures and the efficiency of intramolecular energy transfer (ET), we have successfully developed a methodology for preparing molecular architectures that incorporate energy Donor and energy Acceptor moieties as well as spacer moieties, in a controlled way in the polymer backbone. ${ }^{13}$ We have previously demonstrated the importance of Donor-Acceptor geometries that can be modulated through control of oligomer/polymer molecular architecture. ${ }^{11}$ Through SFRP in the current study we have thus prepared a series of oligomers/polymers of controlled molecular weights, and relatively low polydispersities. The present flexible approach allows us to incorporate various Donor-Acceptor moieties in order to explore the strong reducing potential of various Donor excited states.

In the present report we have successfully synthesized azo-acenaphthylene oligomers 4 with $n$ values up to 4, 5, 7, 17 and 19 via nitroxide mediated polymerization (NMP) using TEMPO as the capping agent for control of the growing polymer chains. Block copolymers, 5, (Scheme 4) with 4-(naphthalenemethoxy)styrene as a vinyl monomer were synthesized using $\mathbf{4}$ as the initiator oligomer. Characterization of the oligomers and copolymers was accomplished using MALDI-MS supported by GPC and ${ }^{1} \mathrm{H}$ NMR spectrometry. MALDI-MS afforded definitive results by providing an inter-peak interval of $152(\mathrm{~m} / \mathrm{z})$, corresponding to acenaphthylene monomer, and inter-peak interval of 260 $(\mathrm{m} / \mathrm{z})$ for the 4-(naphthalenemethoxy)styrene repeating unit in block copolymers, 5. Future studies will be directed to synthesis of copolymers comprising both rigid/stiff and soft/ coil monomeric segments with a view to probing their effect on light harvesting properties.

Acknowledgments. We thank NSERC Canada (E.B) and HEC Pakistan (D.A) for support. Dildar Ali is especially thankful to Higher Education Commission of Pakistan for a fellowship under the Split Ph.D. Program. Support by Vice President's Research Fund of Grenfell Campus, Memorial University of Newfoundland (formerly Sir Wilfred Grenfell College) (J.M.D.) is also noted.

Supplementary Information. It is available through the
Bulletin of the Korean Chemical Society.

\section{References}

1. Balzani, V.; Credi, A.; Venturi, M. Org. Nanostruct. 2008, 1-31.

2. Chu, C. C.; Bassani, D. M. Photochem. \& Photobiol. Sci. 2008, 7, 521-530.

3. Trenor, S. R.; Schultz, A. R.; Love, B. J.; Long, T. E. Chem. Rev. 2004, 104, 3059-3077.

4. Bredas, J. L.; Beljonne, B.; Coropceanu, V.; Cornil, J. Chem. Rev. 2004, 104, 4971-5003.

5. Chen, M.; Ghiggino, K. P.; Thang, S. H.; Wilson, G. J. Polym. Int. 2006, 5, 757-763.

6. Adronov, A.; Gilat, S. L.; Fréchet, J. M. J.; Ohta, K.; Neuwahl, F. V. R.; Fleming, G. R. J. Am. Chem. Soc. 2000, 122, 1175-1185.

7. Webber, S. E. Chem. Rev. 1990, 90, 1469-1482.

8. Abdallah, D.; Ghani, M. A. A.; Cunningham, M. F.; Kazmaier, P. M.; Keoshkerian, B.; Buncel, E. Can. J. Chem. 2004, 82, $1393-$ 1402.

9. Cheon, K. S.; Kazmaier, P. M.; Keum, S. R.; Park, K. T.; Buncel, E. Can. J. Chem. 2004, 82, 551-566.

10. Ghani, M. A. A.; Abdallah, D.; Kazmaier, P. M.; Keoshkerian, B.; Buncel, E. Can. J. Chem. 2004, 82, 1403-1412.

11. Abdallah, D.; Whelan, J.; Dust, J. M.; Hoz, S.; Buncel, E. J. Phys. Chem. A 2009, 113, 6640-6647.

12. Guillet, J. E. Pure Appl. Chem. 1991, 63, 917-924.

13. Ali, D.; Ahmed, Z.; Kazmaier, P. M.; Buncel, E. Can. J. Chem. 2010, $88,910-921$.

14. Hawker, C. J. Acc. Chem. Res. 1997, 30, 373-384.

15. Hawker, C. J.; Bosman, A. W.; Harth, E. Chem. Rev. 2001, 101, 3661-3688.

16. Veregin, R. P. N.; Georges, M. K.; Hamer, G. K.; Kazmaier, P. Trends in Polym. Sci. 1994, 2, 66-72.

17. (a) Sawamoto, M.; Kamigaito, M. Trends in Polym. Sci. 1996, 4, 371-377. (b) Uegaki, H.; Kotani, Y.; Kamigaito, M.; Sawamoto, M. Macromolecules 1997, 30, 2249-2253. (c) Fuuzaki, Y.; Tomita, Y., Terashima, T.; Ouchi, M.; Sawamoto, M. Macromolecules 2010, 43, 5989-5995.

18. Lowe, A. B.; Summerlin, B. S.; Donovan, M. S.; Thomas, D. B.; Hennaux, P.; McCormick, C. L. Advances in Controlled/Living Radical Polymerization; American Chemical Society Symposium Series 854; Matyjaszewski, K., Ed.; American Chemical Society: Washington, D.C., 2003; pp 586-602.

19. McCormick, C. L.; Lowe, A. B. Acc. Chem. Res. 2004, 37, $312-$ 325.

20. Chen, M.; Ghiggino, K. P.; Mau, A. W. H.; Rizzardo, E.; Sasse, W. H. F.; Thang, S. H.; Wilson, G. J. Macromol 2004, 37, 5479-5481.

21. Chen, M.; Ghiggino, K. P.; Rizzardo, E.; Thang, S. H.; Wilson, G. J. Chem. Commun. 2008, 1112-1114.

22. Chen, M.; Ghiggino, K. P.; Mau, A. W. H.; Rizzardo, E.; Thang, S. H.; Gilson, G. J. Chem. Commun. 2002, 2276-2277.

23. Chen, L. H.; Ghiggino, K. P.; Mau, A. W. H.; Sasse, W. H. F.; Thang, S. H.; Wilson, G. J. Macromol 2005, 38, 3475-3481.

24. Pasch, H.; Schrepp, W. MALDI-TOF Mass Spectrometry of Synthetic Polymer; Springer: New York, N.Y. 2003.

25. Perrin, D. D.; Armarego, W. L. F. Purification of Laboratory Chemicals, $3^{\text {rd }}$ ed; Pergamon Press: Toronto, Canada. 1988.

26. Dust, J. M.; Arnold, D. R. J. Am. Chem. Soc. 1983, 105, 12211227.

27. Gopalan, P.; Zhang, Y.; Li, X.; Wiesner, U.; Ober, C. K. Macromol. 2003, 36, 3357-3364.

28. Gopalan, P.; Li, X.; Li, M.; Ober, C. K.; Gonzales, C. P.; Hawker, C. J. J. Polym. Sci: Part A: Polym. Chem. 2003, 41, 3640-3656. 